\title{
Micro-arteriovenous fistula in patients with lower limb lymphedema
}

\author{
Hikaru Kono ${ }^{1}$, Hisashi Sakuma ${ }^{2}$, Shiho Watanabe ${ }^{3}$, Takaya Murayama ${ }^{4}$, Masashi Takemaru ${ }^{5}$ \\ ${ }^{1}$ Department of Plastic and Reconstructive Surgery, Tachikawa Hospital, Tachikawa; ${ }^{2}$ Department of Plastic and Reconstructive Surgery, \\ Ichikawa General Hospital, Ichikawa; ${ }^{3}$ Department of Plastic and Reconstructive Surgery, Keio University Hospital, Tokyo; ${ }^{4}$ Department of \\ Vascular Surgery, Murayama Clinic, Yokohama; Department of Plastic and Reconstructive Surgery, Yokohama Municipal Citizen's Hospital, \\ Yokohama, Japan
}

Background A micro-arteriovenous fistula (AVF) is a minute, short shunt between an artery and a vein that does not pass through a capillary. We investigated the association between micro-AVFs and lymphedema using computed tomography angiography (CTA) and venous blood gas analysis.

Methods In 95 patients with lower limb lymphedema, the presence or absence of early venous return (EVR) was compared between patients with primary and secondary lymphedema. Furthermore, we investigated the difference in the timing of edema onset in patients with secondary lymphedema with or without EVR using CTA. In 20 patients with lower limb lymphedema with confirmed early EVR in a unilateral lower limb, the partial pressure of oxygen $\left(\mathrm{PO}_{2}\right)$ was compared between the lower limb with EVR and the contralateral lower limb.

Results Secondary lymphedema with or without EVR occurred at an average of $36.0 \pm 59.3$ months and $93.5 \pm 136.1$ months, respectively; however, no significant difference was noted. $\mathrm{PO}_{2}$ was $57.6 \pm 11.7 \mathrm{mmHg}$ and $44.1 \pm 16.4 \mathrm{mmHg}$ in the EVR and non-EVR limbs, respectively, which was a significant difference $(\mathrm{P}=0.005)$.

Conclusions EVR and venous blood gas analysis suggested the presence of micro-AVFs in patients with lower extremity edema. Further research is warranted to examine the cause of micro-AVFs, to advance technology to facilitate the confirmation of micro-AVFs by angiography, and to improve lymphedema by ligation of micro-AVFs.

Keywords Lymphedema / Arteriovenous fistula / Venous insufficiency / Venous hypertension / Computed tomography angiography

Received: August 24, 2020 • Revised: November 1, 2020 • Accepted: November 11, 2020

pISSN: 2234-6163 • elSSN: 2234-6171 • https://doi.org/10.5999/aps.2020.01704 • Arch Plast Surg 2021;48:219-223
Correspondence: Hisashi Sakuma Department of Plastic and Reconstructive Surgery, Ichikawa General Hospital, 5-11-13, Sugano, Ichikawa 272-8513, Japan Tel: +81-473-22-0151 Fax: +81-473-25-4456 E-mail: prssakuma@gmail.com

\section{INTRODUCTION}

A micro-arteriovenous fistula (AVF) is a minute, short shunt between an artery and a vein that does not pass through a capillary. In 1995, Haimovici [1] defined a micro-AVF as a vessel with a pulsatile waveform without a dicrotic notch on ultraso- nography, which passes into a saphenous vein and a perforator vein, with a diameter of approximately $0.1-2.0 \mathrm{~mm}$. Recently, some reports have suggested that the surgical ligation of microAVFs or incompetent perforator veins due to micro-AVFs could improve intractable edema, skin redness, pain, and ulceration of the lower limbs [2-4]. 
Among patients with lower limb lymphedema, we often encounter patients with edema between the lower thigh and the dorsum, associated with redness, heat, and pain, without an increased inflammatory response in blood tests. In such cases, there is no evidence of phlebostasis, such as deep venous thrombosis (DVT), and the effect of lymphaticovenular anastomosis (LVA) is not satisfactory. It is presumed that micro-AVFs are one of the reasons for this condition, but there are few reports on the association between lymphedema and micro-AVFs [5].

We performed computed tomography angiography (CTA) in patients with lower limb lymphedema and determined the presence or absence of early venous return (EVR), as indicated by early contrast filling in the dilated lower limb superficial veins during the arterial phase. Through this assessment, the potential correlation between EVR and micro-AVFs was investigated.

\section{METHODS}

We performed CTA in 95 patients ( 32 with primary lymphedema and 63 with secondary lymphedema) who visited our department because of lower limb lymphedema between June 2017 and May 2018. Using blood tests, ultrasonography, and computed tomography (CT), we excluded patients with DVT. The clinical features of the cases are shown in Table 1. The protocol for this study was approved by the relevant institutional review board (IRB No. 19-11-01), and all authors adhered to the STROBE guidelines. All patients provided written informed consent to the publication of this article.

The patients with primary lymphedema $(\mathrm{n}=32)$ had congenital lymphedema $(n=2)$, lymphedema praecox $(n=6)$, or lymphedema tarda $(\mathrm{n}=24)$. The 63 patients with secondary lymphedema had undergone surgery for cervical cancer $(\mathrm{n}=$ $20)$, endometrial cancer $(n=27)$, ovarian cancer $(n=4)$, vaginal cancer $(n=1)$, bladder cancer $(n=2)$, prostate cancer $(n=3)$, rectal cancer $(n=2)$, duodenal cancer $(n=1)$, or uterine cancer

Table 1. Clinical features of patients

\begin{tabular}{lcc}
\hline Variable & $\begin{array}{c}\text { Secondary } \\
\text { lymphedema }\end{array}$ & $\begin{array}{c}\text { Primary } \\
\text { lymphedema }\end{array}$ \\
\hline No. of cases & 63 & 32 \\
Sex (male/female) & $7 / 56$ & $9 / 23$ \\
Median age (range) (yr) & $65(38-88)$ & $59(18-89)$ \\
Complications & & \\
$\quad$ Hypertension & 13 & 10 \\
Dyslipidemia & 12 & 2 \\
Diabetes mellitus & 7 & 5 \\
Ischemic heart disease & 2 & 0 \\
Congestive heart disease & 1 & 1 \\
Chronic kidney disease & 0 & 0 \\
Chronic obstructive pulmonary disease & 0 & 0 \\
\hline
\end{tabular}

$(\mathrm{n}=3)$. The three latter cases were described simply as uterine cancer and therefore could not be classified more specifically as endometrial cancer or cervical cancer.

First, we investigated the difference in the presence or absence of EVR among those with primary and secondary lymphedema, the difference in timing of edema onset among those with secondary lymphedema who did or did not have EVR, and the difference in body mass index (BMI) among those with or without EVR. For secondary lymphedema, the presence or absence of iliac vein stenosis was also examined.

Thereafter, we compared the partial pressure of oxygen $\left(\mathrm{PO}_{2}\right)$ in 20 patients who underwent venous blood gas analysis of both lower limbs and were confirmed through CTA as having EVR in a unilateral lower limb. Ultrasound guidance was used for both the EVR and non-EVR limbs to identify and draw blood from the giant saphenous vein, which had the largest vascular diameter. Blood was collected under room air, with the patient in a supine position.

We used the Student $t$-test, with a P-value $<0.05$ considered to indicate statistical significance, to analyze the timing of the onset of edema, BMI, and $\mathrm{PO}_{2}$. The Fisher exact test was used to evaluate the significance of differences in the presence or absence of EVR among those with primary and secondary lymphedema, with the threshold for statistical significance set at $\mathrm{P}<0.05$. All data are expressed as mean \pm standard deviation.

\section{RESULTS}

CT revealed early contrast filling in the dilated superficial veins during the arterial phase, indirectly indicating the presence of micro-AVFs (Fig. 1).

EVR was seen in $59.4 \%$ (19 of 32) of patients with primary lymphedema and in $46.0 \%$ ( 29 of 63 ) of those with secondary lymphedema, but no significant difference was observed be-
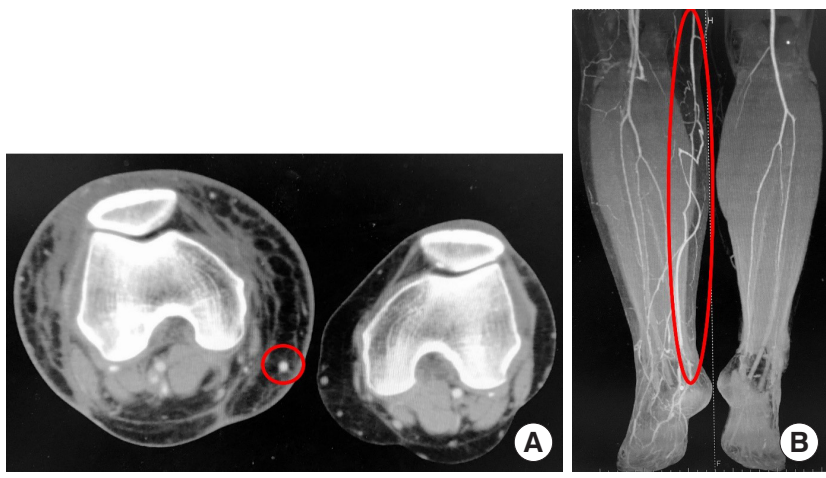

Fig. 1. Early venous return. $(A, B)$ Computed tomography angiography showing early venous return. The red circle is the great saphenous vein. The images show observations in the arterial phase. 
Table 2. Proportion of the presence or absence of EVR in secondary and primary lymphedema

\begin{tabular}{lccc}
\hline Presence of EVR & $\begin{array}{c}\text { Secondary } \\
\text { lymphedema } \\
(\mathrm{n}=63)\end{array}$ & $\begin{array}{c}\text { Primary } \\
\text { lymphedema } \\
(\mathrm{n}=32)\end{array}$ & P-value \\
\hline No. (\%) & $29(46.0)$ & $19(59.4)$ & 0.279 \\
\hline
\end{tabular}

EVR, early venous return.

Table 3. Onset of edema in secondary lymphedema with and without EVR

\begin{tabular}{lccc}
\hline Onset of edema (mon) & $\begin{array}{c}\text { EVR }(+) \\
(n=26)\end{array}$ & $\begin{array}{c}\text { EVR }(-) \\
(n=31)\end{array}$ & P-value \\
\hline Mean \pm SD & $36.0 \pm 59.3$ & $93.5 \pm 136.1$ & 0.051 \\
\hline
\end{tabular}

EVR, early venous return.

tween the groups $(\mathrm{P}=0.279)$ (Table 2).

Regarding the onset of edema, data were obtained from $26 \mathrm{pa}-$ tients with EVR and 31 patients without EVR who had secondary lymphedema. Secondary lymphedema with or without EVR occurred at an average of $36.0 \pm 59.3$ months and $93.5 \pm$ 136.1 months, respectively, but no significant difference was detected $(\mathrm{P}=0.051)$ (Table 3$)$.

BMI were obtained from 30 patients with EVR and 28 patients without EVR. The BMI of patients with lower limb lymphedema with and without EVR was $26.4 \pm 6.20 \mathrm{~kg} / \mathrm{m}^{2}$ and $25.6 \pm 7.39 \mathrm{~kg} / \mathrm{m}^{2}$, respectively, which was not a significant difference $(\mathrm{P}=0.692)$ (Table 4$)$.

The distribution of iliac vein stenosis was almost identical in both groups, as it was found in three of the 48 patients with EVR and two of the 47 patients without EVR. Blood gas analysis revealed that $\mathrm{PO}_{2}$ was significantly higher in the EVR limbs (57.6 \pm $11.7 \mathrm{mmHg})$ than in the non-EVR limbs $(44.1 \pm 16.4 \mathrm{mmHg})$ $(\mathrm{P}=0.005)$ (Table 5).

\section{DISCUSSION}

In this study, we performed CTA in patients with lower limb lymphedema to determine the presence or absence of EVR, as detected by early contrast filling in the dilated superficial veins of the lower limb during the arterial phase. This was thought to indicate that arterial blood was flowing into the venous blood at an early phase, suggesting the presence of a shunt between the arteries and veins. However, we could not discover any shunts on CTA; therefore, we surmised that these patients may have had thin shunts, or micro-AVFs, which are difficult to identify on images.

The presence of a micro-AVF, which is a minute and short circuit, is difficult to visualize on imaging. No AVFs could be confirmed on CTA and the superficial veins were visualized in the
Table 4. BMI of patients with lower limb lymphedema with and without EVR

\begin{tabular}{lccc}
\hline BMI $\left(\mathrm{kg} / \mathrm{m}^{2}\right)$ & $\begin{array}{c}\text { EVR }(+) \\
(\mathrm{n}=30)\end{array}$ & $\begin{array}{c}\text { EVR }(-) \\
(\mathrm{n}=28)\end{array}$ & P-value \\
\hline Mean \pm SD & $26.4 \pm 6.2$ & $25.6 \pm 7.4$ & 0.692 \\
\hline
\end{tabular}

BMI, body mass index; EVR, early venous return.

Table 5. $\mathrm{PO}_{2}$ in limbs with and without EVR

\begin{tabular}{lccc}
\hline $\mathrm{PO}_{2}(\mathrm{mmHg})$ & $\begin{array}{c}\mathrm{EVR}(+) \\
(\mathrm{n}=20)\end{array}$ & $\begin{array}{c}\mathrm{EVR}(-) \\
(\mathrm{n}=20)\end{array}$ & P-value \\
\hline Mean $\pm \mathrm{SD}$ & $57.6 \pm 11.7$ & $44.1 \pm 16.4$ & $0.005^{\mathrm{a})}$ \\
\hline
\end{tabular}

$\mathrm{PO}_{2}$, partial pressure of oxygen; $\mathrm{EVR}$, early venous return. a) Statistically significant, $\mathrm{P}<0.05$

arterial phase. Additionally, since $\mathrm{PO}_{2}$ was significantly higher in the EVR limbs, arterial blood was considered to be flowing into the veins. This finding was thought to be suggestive of the presence of micro-AVFs. In addition, there was no increased inflammatory response based on blood test results, so it was unlikely that the flow velocity or $\mathrm{PO}_{2}$ within the veins of the lower limbs had increased due to those effects.

An AVF is a non-physiological circulatory shunt between an artery and a vein that does not pass through a capillary. In 1995, Haimovici [1] defined a micro-AVF as a vessel with a pulsatile waveform without a dicrotic notch on ultrasonography, which passes into a saphenous vein and a perforator vein, with its diameter being about $0.1-2.0 \mathrm{~mm}$. Moreover, according to Honda et al. [6], a micro-AVF is approximately $1 \mathrm{~mm}$ or less in diameter and is connected directly to the superficial and perforator veins; pulsatile blood flow through a micro-AVF can be recognized on color-flow Doppler ultrasonography. This pulsatile blood flow is similar to that of an artery and is also recognized on pulse Doppler ultrasonography. A micro-AVF is distinguishable from an AVF, which can be classified as caused by iatrogenic or traumatic factors.

AVFs and micro-AVFs were originally considered as possible causes of varicose veins. In a 1949 report by Pratt [7], AVFs were observed in $24 \%$ of 272 lower-extremity varicose veins. In fact, the oxygen content in varicose veins was reported to be higher than that in the upper-limb veins, the femoral veins of healthy individuals, and the femoral vein on the affected side [8,9]. In 2011, Yoshida and Fujita [10] reported that AVFs were observed on ultrasonography in 10 of 13 people (77\%) after they had bathed in a foot bath, including in healthy individuals.

Recently, some reports have suggested that surgical ligation of micro-AVFs or incompetent perforator veins due to micro-AVFs could improve intractable edema, skin redness, pain, and ulceration of the lower limbs. Muneta et al. [2] reported that they per- 


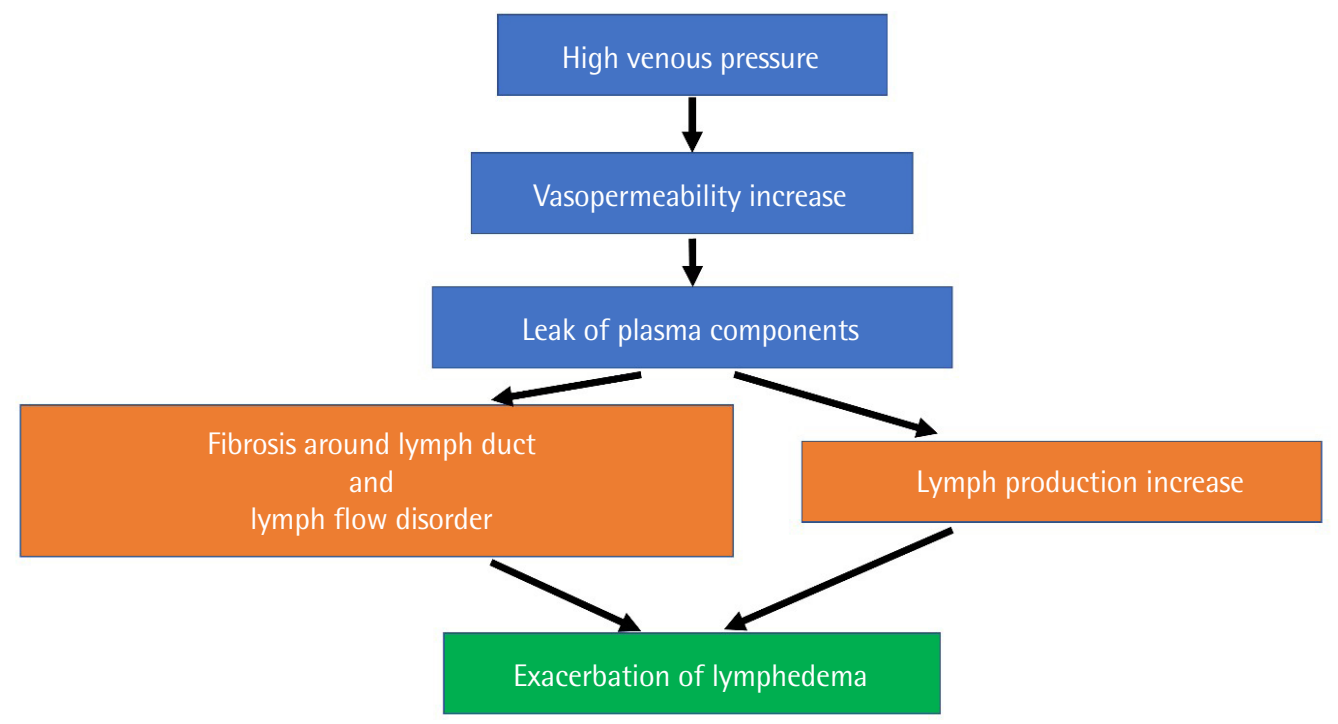

Fig. 2. Hypothetical pathology of lymphedema exacerbation. Micro-arteriovenous fistula increases venous blood pressure owing to the backflow of arterial blood and increases leakage of plasma components. Chronic leakage leads to increased lymph production and fibrosis around the lymph duct.

formed ligation of micro-AVFs, which improved erythema and tenderness of the lower leg. Tanaka et al. [3] and Serizawa et al. [4] reported that micro-AVFs were detected in $13 \%$ of patients who visited their department with edema and skin lesions of the lower limbs, and that patients' symptoms improved following ligation treatment of incompetent perforator veins with arterial pulsatile waveforms on ultrasonography. The micro-AVFs were so thin that they might have been difficult to identify during surgery. However, the perforators connecting with the micro-AVFs became incomplete due to the inflow of arterial blood, and ligation of incompetent perforators with pulsatile waveforms was suggested to be an indirect treatment for micro-AVFs [3]. Sakuma et al. [5] also reported two cases in which ligation of an incompetent perforator with an arterialized waveform on ultrasonography improved secondary limb lymphedema.

Uehara et al. [11] described the treatment for left common iliac vein occlusion and ipsilateral internal iliac AVFs; the peripheral AVF was opened because of the rise in venous pressure due to iliac vein occlusion, and it was presumed that this was one of the causes of swelling and edema. However, in our CT findings, there were few cases of obvious iliac vein narrowing, suggesting that iliac vein occlusion was not a major cause of micro-AVF patency.

It has been reported that CTA was useful as a screening tool for micro-AVFs [3-5]. Since micro-AVFs have small calibers, it is difficult to accurately determine their presence via ultrasonography or arteriography. CT revealed early contrast filling in the dilated superficial veins during the arterial phase, indirectly indicating the presence of micro-AVFs.

In our study, we found that when EVR was present in a lower limb with lymphedema, $\mathrm{PO}_{2}$ was significantly higher in the EVR limb than in the contralateral limb; hence, it is suggested that micro-AVFs were present in the lower limbs of lymphedema patients. An earlier report suggested that ligation of incomplete perforators due to micro-AVFs improved limb lymphede$\mathrm{ma}$, and we therefore presumed that micro-AVFs might be associated with edema. Moreover, although the onset of edema in the EVR limbs was not significantly different from that in the non-EVR limbs, the EVR limbs tended to have an earlier onset time than the non-EVR limbs, which was consistent with the clinical course.

Regarding our hypothesis of the pathology through which a micro-AVF exacerbates lymphedema, it is thought that a persistent increase in venous pressure occurs due to the backflow of arterial blood, resulting in dysfunction of the venous valves, an increase in capillary permeability, and leakage of plasma components. Dysfunctional lymph flow due to fibrosis around the lymph duct and overflow due to lymph production are thought to be two pathologies involved in lymphedema exacerbation (Fig. 2). Takase et al. [12] reported that AVF-induced chronic elevation of venous pressure in a rat model was associated with an inflammatory reaction in venous valves, a process that may have led to their dysfunction, reflux, and upstream elevation of venous pressure.

Especially in the case of lymphatic flow disorder caused by pelvic lymphadenectomy and radiotherapy in secondary lymphedema, the presence of a micro-AVF increases lymphatic production, leading to an additional overload on the lymphatic duct. It is presumed that this overload accelerates the onset and 
deterioration of edema.

Currently, the surgical treatment of lymphedema involves LVA and vascularized lymph node transfer, which is thought to treat the disorder of lymph flow. However, in addition to the conditions listed above, intravenous leakage and excessive production of lymph fluid result from venous hypertension caused by a micro-AVF. Therefore, we believe that a suitable treatment, such as ligation of a micro-AVF or an incompetent perforator, is also necessary.

The limitation of our study is that it was difficult to distinguish between physiologically functional micro-AVFs and abnormally formed micro-AVFs. Moreover, the theory that micro-AVFs exacerbate lymphedema is only tentative; it is necessary to study cases in which lymphedema improved after the treatment of micro-AVFs.

For future research, many aspects that have not been elucidated, such as the causes of micro-AVFs and the sites where they occur, need to be studied. At our department, we predominantly perform compression therapy and LVA as treatment for lymphedema. However, we have encountered many cases that were not improved by these treatments alone, and it is thought that an increase in venous pressure and hyperpermeability of the capillaries resulting from micro-AVFs might be implicated in these cases as causes of refractory lymphedema. EVR and venous blood gas analysis suggested the presence of micro-AVFs in lower extremity lymphedema.

\section{NOTES}

\section{Conflict of interest}

No potential conflict of interest relevant to this article was reported.

\section{Ethical approval}

The study was approved by the Institutional Review Board of Yokohama Municipal Citizen's Hospital (IRB No. 19-11-01) and performed in accordance with the principles of the Declaration of Helsinki. Written informed consent was obtained.

\section{Patient consent}

The patients provided written informed consent for the publication and the use of their images.

\section{Author contribution}

Conceptualization: H Kono, H Sakuma, S Watanabe, T Murayama, M Takemaru. Data curation: H Kono, H Sakuma, S Watanabe. Formal analysis: H Kono, H Sakuma, M Takemaru. Methodology: H Kono, S Watanabe, T Murayama, M Takema- ru. Visualization: T Murayama. Writing - original draft: H Kono. Writing - review \& editing: S Watanabe, T Murayama, M Takemaru.

\section{ORCID}

Hikaru Kono

https://orcid.org/0000-0001-5736-0312

Hisashi Sakuma

https://orcid.org/0000-0002-6073-6832

Shiho Watanabe

https://orcid.org/0000-0001-5876-7237

Takaya Murayama

https://orcid.org/0000-0003-1895-3777

Masashi Takemaru

https://orcid.org/0000-0001-8346-6989

\section{REFERENCES}

1. Haimovici $H$. Role of A-V shunting in varicose veins: therapeutic implications. J Cardiovasc Surg (Torino) 1995;36: 109-15.

2. Muneta K, Okubo Y, Sawada Y. Sclerosing panniculitis in a patient with arteriovenous fistula of the lower leg and summary of 13 cases. Jpn J Clin Dermat 2017;71:419-23.

3. Tanaka M, Serizawa F, Nagaoka Y, et al. Two cases of microarteriovenous fistula in the lower extremity with misdiagnosis of refractory cellulitis. Ann Vasc Dis 2018;11:148-52.

4. Serizawa F, Tanaka M, Shimizu T, et al. The epidemiology of micro-arteriovenous fistulas in the lower legs. Ann Vasc Surg 2019;56:29-35.

5. Sakuma H, Watanabe S, Kono H. A novel surgical approach for refractory secondary lymphedema of the lower extremity accompanying micro-arteriovenous fistula. Ann Vasc Surg 2020;65:284.

6. Honda K, Komai H, Juri M. Micro-arteriovenous fistula of the lower extremities with severe skin lesions. J Jpn Coll Angiol 2006;46:73-8.

7. Pratt GH. Arterial varices; a syndrome. Am J Surg 1949;77: 456-60.

8. Blalock A. Oxygen content of blood in patients with varicose veins. Arch Surg 1929;19:898-905.

9. Fontaine R. Remarks concerning venous thrombosis and its sequelae. Surgery 1957;41:6-25.

10. Yoshida Y, Fujita M. Shunt flow of arteriovenous fistulas from plantar artery. Phlebology 2011;26:32-4.

11. Uehara A, Yamamoto K, Mishima T, et al. A case of venous reconstruction for common iliac vein occlusion and ipsilateral internal iliac arteriovenous fistulae. Jpn J Vasc Surg 2008;17:575-80.

12. Takase S, Pascarella L, Lerond L, et al. Venous hypertension, inflammation and valve remodeling. Eur J Vasc Endovasc Surg 2004;28:484-93. 